Mathematical Modeling

\title{
STABILIZATION OF ELASTIC INVERTED PENDULUM WITH HYSTERESIS
}

\author{
M.E. Semenov ${ }^{1}$, A.M. Solovyev ${ }^{2}$ \\ ${ }^{1}$ Voronezh State University, Voronezh, Russia \\ ${ }^{2}$ JSC Concern "Sozvezdie", Voronezh, Russia
}

\begin{abstract}
In this article, we investigate an elastic inverted pendulum with hysteretic nonlinearity (a backlash) in a suspension point. Namely, problems of stabilization and the optimization of such a system are considered. An algorithm (based on a bionic model) which provides effective procedure for finding of optimal parameters is presented and applied to considered system. The results of numerical simulations, namely the phase portraits and the dynamics of Lyapunov function are also presented and discussed.
\end{abstract}

Keywords: elastic inverted pendulum, hysteretic control, stabilization problem, bionic algorithm.

Citation: Semenov ME, Solovyev AM. Stabilization of elastic inverted pendulum with hysteresis. CEUR Workshop Proceedings, 2016; 1638: 650-657. DOI: 10.18287/1613-0073-2016-1638-650-657

\section{Introduction}

As known, the problem of the inverted pendulum plays a central role in the control theory [1-11]. In particular, the problem of inverted pendulum (as a test model) provides many challenging problems to control design. Because of their nonlinear nature pendulums have maintained their usefulness and they are now used to illustrate many of ideas emerging in the field of nonlinear control [12]. Typical examples are feedback stabilization, variable structure control, passivity-based control, back-stepping and forwarding, nonlinear observers, friction compensation, and nonlinear model reduction. The challenges of control made the inverted pendulum systems a classic tool in control laboratories. It should also be noted that the problem of stabilization of such a system is a classical problem of the dynamics and the control theory. Moreover, the model of inverted pendulum is widely used as a standard for testing of the control algorithms (for PID controller, neural networks, fuzzy control, etc.).

According to control purposes of the inverted pendulum, the control of inverted pendulum can be divided into three aspects. The first widely researched aspect is the swing-up control of the inverted pendulum [13-15]. The interesting and important results on the time optimal control of the inverted pendulum were obtained in [13, 15]. In particular, in [15], the optimal transients (taking into account the cylindrical 
character of the state space of the system under control) were built for different values of the parameters and constraints on the control torque. The second aspect is the stabilization of the inverted pendulum $[16,17]$. The third aspect is the tracking control of the inverted pendulum [18].

In practice, stabilization and tracking control are more useful for an application. A backlash in the suspension point is a kind of hysteretic nonlinearity. The hysteretic phenomena (especially in the form of control parameters) play an important role in such a fields as physics, chemistry, biology, economics, etc. It should also be pointed out that the hysteretic phenomena are insufficiently known in our days. The purpose of this paper is investigation of the possible stabilization (in a vicinity of vertical position) of the elastic inverted pendulum in the presence of a backlash in the suspension point together with investigation of various aspects of such a dynamical system.

\section{Problem}

Let's consider the model of stabilization of the inverted pendulum in the vicinity of the vertical position. The pendulum is considered as an elastic rod which is hingedly fixed on a cylinder. Motion of the cylinder is excited by the horizontal motion of a piston (see the Fig. 1).

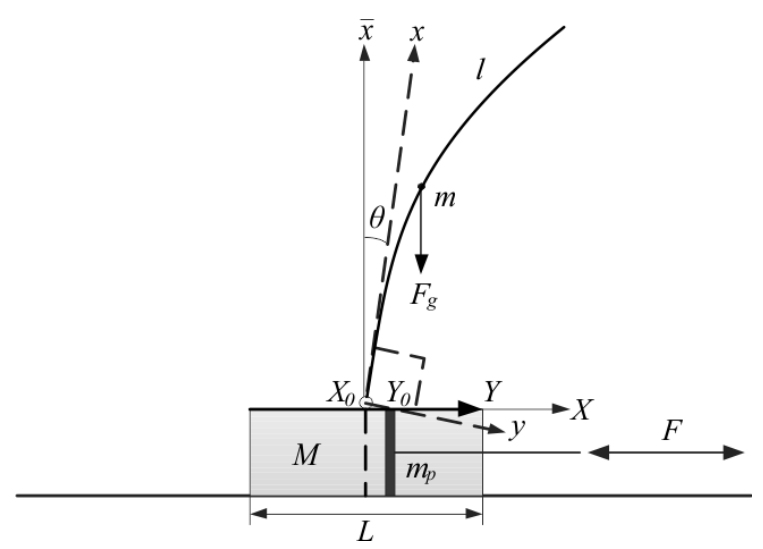

Fig. 1. Model of elastic inverted pendulum: geometry of the problem

A mathematical model of a similar mechanical system was considered in [19]. Investigation of the dynamics of an elastic inverted pendulum was carried out in [20-23]. Here $(x, y)$ is the coordinates of the elastic rod with mass $\mathrm{m}$ and density $\rho$; the $O x$ axis coincides with a tangent to rod's profile in the suspension point; $\theta$ is an angle of slope for the coordinates of a rod, and $I$ is a centroidal moment of inertia of the rod's section;

$(X, \bar{x})$ is the Cartesian coordinate system connected with a considered mechanical system (namely the $X$ coordinate determines the position of the piston in a cylinder), 
$M$ is a mass of a cylinder with length $L$, and $F$ is a force joined to the piston with mass $m_{p}$ (such a force is treated as control).

\section{Hysteretic nonlinearity}

In the following consideration, we use the operator technique for the hysteretic nonlinearities following the ideas of Krasnosel'skii and Pokrovskii [24]. Output of the backlash operator on the monotonic inputs can be described by the following expression:

$$
\begin{aligned}
& X(t)=\Gamma\left[X_{0}, L\right] \cdot Y(t)= \\
& =\left\{\begin{array}{l}
0, \quad\left|Y(t)-X_{0}\right| \leq \frac{L}{2}, \\
Y(t)-\frac{L}{2}, \quad Y(t)-X_{0}>\frac{L}{2}, \\
Y(t)+\frac{L}{2}, \quad Y(t)-X_{0}<-\frac{L}{2} .
\end{array}\right.
\end{aligned}
$$

Here $X_{0}$ is the initial position of the piston in a cylinder. Such an expression (action of such an operator) can be illustrated by the Fig. 2 .

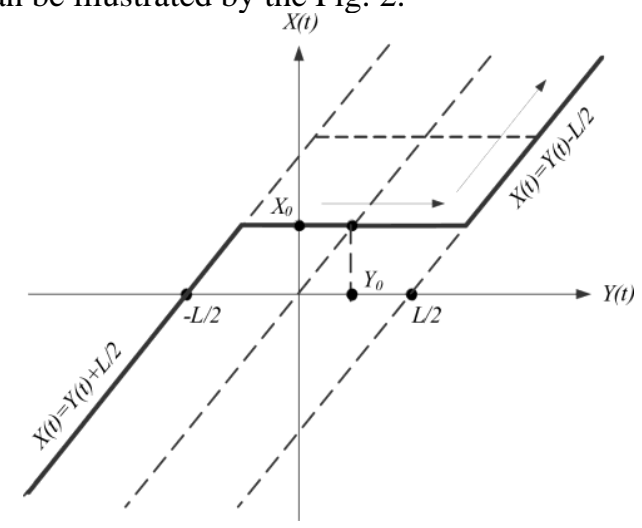

Fig. 2. Dynamics of input-output relation for the backlash operator

The detailed description of this operator as well as its properties is considered in the book of Krasnosel'skii and Pokrovskii [24].

Here $X(t)$ is a displacement of the cylinder's center, and $Y(t)$ is a displacement of the piston in the horizontal plane (see Fig. 1).

\section{Physical model}

Let's assume that the deviation $y$ and angle $\vartheta$ are small, i.e., $x \approx \bar{x}$ and the boundary conditions that determine the curvature of the pendulum are: 
$\left\{\begin{array}{l}y(0, t)=y^{\prime \prime}(0, t)=0 \\ y^{\prime \prime}(l, t)=y^{\prime \prime \prime}(l, t)=0\end{array}\right.$

The function $X(\bar{x}, t)$ describes the behavior of the pendulum's profile in the time and shows the deviation of the pendulum's points relative to the vertical axis; $(X, \bar{x})$ are the coordinates of the pendulum's profile, and $X(0, t)=s(t)$ is a displacement of the suspension point in the horizontal plane.

The coordinate system transformation in the matrix form is given by $\left(\begin{array}{l}X \\ \bar{x}\end{array}\right)=\left(\begin{array}{cc}\cos \theta & \sin \theta \\ -\sin \theta & \cos \theta\end{array}\right)\left(\begin{array}{l}y \\ x\end{array}\right)+\left(\begin{array}{c}X(0, t) \\ 0\end{array}\right)$

Let us construct the physical model of the considered mechanical system taking into account a backlash in the suspension point of the elastic rod. In order to do this, we use the Lagrange formalism.

Making the same transformations (Lagrange formalism, variational principle and Tailor's expansion), the system of equations which describes the dynamics of the system under consideration has the following form:

$$
\left\{\begin{array}{l}
\ddot{X}+\frac{E I l}{m} X^{\prime \prime \prime \prime}=g X^{\prime}(0, t), \\
(M+m) \ddot{X}(0, t)+m l \ddot{X}^{\prime}(0, t)=f(t), \\
\frac{g}{l}(M+m) X(0, t)-\frac{M E I}{m} X^{\prime \prime \prime}(0, t)=f(t), \\
f(t)=\Gamma\left[X(0, t), Y(t), L, F_{0}\right] F, \\
m_{p} \ddot{Y}(t)=F .
\end{array}\right.
$$

\section{Stabilization}

Let us consider the problem of control of the pendulum using the feedback principles, i.e., the force which affects the piston can be presented by the following equality:

$F=k \cdot \operatorname{sign}\left(a \cdot e_{1}+e_{2}\right)$,

where $\mathrm{a}>0, k>0$ and

$\left\{\begin{array}{l}e_{1}=\int_{0}^{l} X^{\prime} d l, \\ e_{2}=\int_{0}^{l} \dot{X}^{\prime} d l .\end{array}\right.$

Here $e_{1}$ is an average angle of the rod's deviation, and $e_{2}$ is an average angular velocity of the rod. 


\section{Optimization problem}

As was mentioned above, the problem's solution on stabilization of the elastic inverted pendulum in the vicinity of the upper position is consisted in search of the optimal values for coefficients $a$ and $k$.

In order to solve the optimization problem in the system under consideration, we use the bionic adaptation algorithms because the hysteretic peculiarities in the considered pendulum's model lead to some difficulties in use of the classical optimization algorithms due to nondifferentiability of the functions in the system of equations.

Such algorithms are the part of the line of investigation that can be called as an "adaptive behavior." Main method of this line consists in the investigation of artificial organisms (in a form of a computer program or a robot) that can be named as animats (these animats can be adapted to the environment). The behavior of animats emulates the behavior of animals.

Actual line of investigation in the frame of the animat approach is emulation of searching behavior of animals $[25,26]$. Let us consider the bionic model of adaptive searching behavior on the example of caddis-flies larvae or Chaetopteryx villosa. The main schema of searching behavior can be characterized by the two stages:

Motion in chosen direction (conservative tactics);

Random change of motion direction (stochastic searching tactics).

We consider this model for the simple case of maximum search for the function of two variables. Let us describe the main stages of the considered model:

1. We consider an animat which is moved in the twodimensional space $x, y$. Main purpose of animat is maximum search for the function $\mathrm{f}(\mathrm{x}, \mathrm{y})$.

2. Animat is functioned in discrete time $t=0,1,2, \ldots$ Animat estimates the change of current value of $\mathrm{f}(\mathrm{x}, \mathrm{y})$ in comparison with the previous time

$\Delta f(t)=f(t)-f(t-1)$

3. Every time animat moves so its coordinates $\mathrm{x}$ and $\mathrm{y}$ change by $\Delta \mathrm{x}(\mathrm{t})$ and $\Delta \mathrm{y}(\mathrm{t})$, respectively.

4. Animat has two tactics of behavior:

a) conservative tactics;

b) stochastic searching tactics.

In that way, we can use the proposed algorithm for searching the optimal control in the stabilization problem of the elastic inverted pendulum. Taking into account the reasoning presented above, we can apply the presented algorithm to functional $\mathrm{J}(\mathrm{a}, \mathrm{k})$ where the coefficients a and $\mathrm{k}$ determine the character of control of the mechanical. Due to the fact that the presented bionic algorithm is used to maximum search of the function of two variables, we will consider minimization of the functional as procedure for finding the coefficients a and $\mathrm{k}$ that lead to realization of the condition $-J(a, k) \rightarrow \max$. 


\section{Simulation results}

Characteristics and initial conditions for the mechanical system under consideration are: $m=1 \mathrm{~kg} ; M=10 \mathrm{~kg} ; l=1 \mathrm{~m} ; \rho=1,04 \mathrm{~kg} / \mathrm{m} ; E=210 \cdot 10^{9} \mathrm{~Pa} ; I=0,087 \mathrm{~kg} \cdot \mathrm{m}^{2} ; \alpha=$ $0.06^{\circ} ; L=0,01 \mathrm{~m} ; m_{p}=1 \mathrm{~kg}$.

In the searching process for optimization (using the bionic algorithm), we have obtained the following values of coefficients: $\mathrm{a}=8,4$ and $\mathrm{k}=1,39$.

In order to estimate the stability of the considered system, we use the Lyapunov criterion. In particular, we use the following Lyapunov function:

$V=e_{1}^{2}+e_{2}^{2}$.

The phase trajectory of such a system together with the dynamics of Lyapunov function in time (namely in discrete time which corresponds to the difference scheme) are presented in the Fig. 3. In this figure, the integral angle $e_{1}$ and integral angular velocity $e_{2}$.
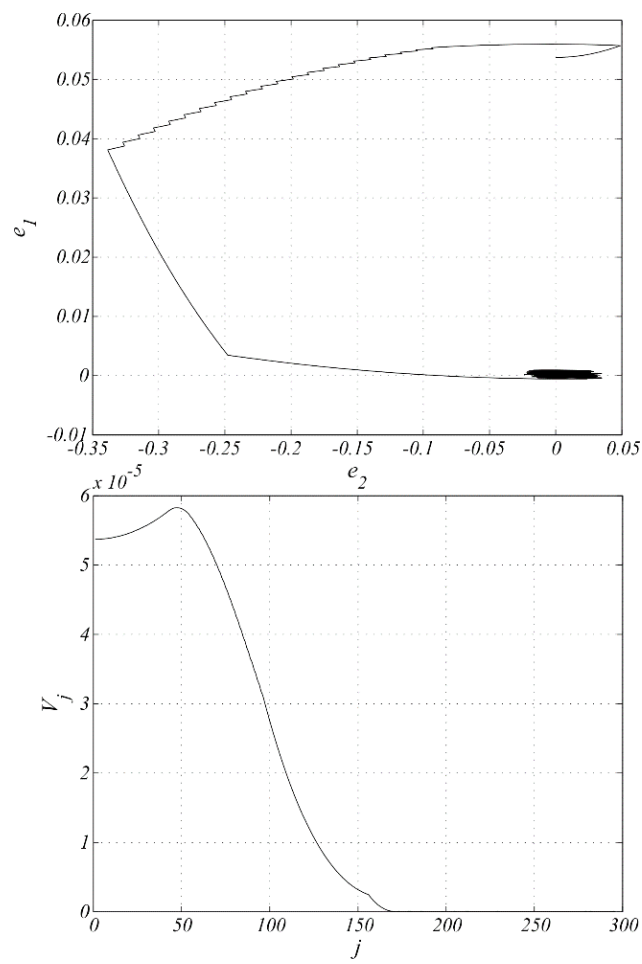

Fig. 3. Phase trajectory (top panel) and dynamics of Lyapunov function (bottom panel)

\section{Conclusions}

In this paper, we have considered the stabilization problem of the elastic inverted pendulum under hysteretic control in the form of a backlash in the suspension point. 
Also, the problem of the optimization for the system under consideration was analyzed. Main coefficients, namely $a$ and $k$, that provide the solution of the optimization problem for the considered system were obtained using the so-called bionic algorithm.

All the results on stabilization of the system under consideration have obtained using the corresponding numerical methods based on the difference scheme. The results of numerical simulations show that the considered system eventually tends to the stable state both in the case of the absence of a backlash and in the case of its presence. These facts were presented in the form of the corresponding phase portraits for the considered system. Moreover, in order to estimate the stability of the elastic pendulum with the hysteretic nonlinearity in the suspension point, we have used the Lyapunov criterion and the dynamics of the corresponding Lyapunov function has also been presented.

\section{Acknowledgement}

The work was partially funded by the Russian Foundation for Basic Research Grants (project №16-08-00312).

\section{References}

1. Arkhipova IM, Luongo A. Stabilization via parametric excitation of multi-dof statically unstable systems. Commun. Nonlinear Sci. Numer. Simul, 2014; 19: 3913-3926.

2. Boubaker O. The inverted pendulum: a fundamental benchmark in control theory and robotics. International Conference on Education and e-Learning Innovations (ICEELI 2012), 2012: 1-6.

3. Butikov EI. Subharmonic resonances of the parametrically driven pendulum. J. Phys. A Math. Theor., 2002; 35: 6209.

4. Chernous'ko FL, Reshmin SA. Time-optimal swing-up feedback control of a pendulum. Nonlinear Dynam, 2007; 47: 65-73.

5. Huang J, Ding F, Fukuda T, Matsuno T. Modeling and velocity control for a novel narrow vehicle based on mobile wheeled inverted pendulum. IEEE Trans. Control Syst. Technol., 2013; 21: 1607-1617.

6. Kim KD, Kumar P. Real-time middleware for networked control systems and application to an unstable system. IEEE Trans. Control Syst. Technol., 2013; 21: 1898-1906.

7. Mason P, Broucke M, Piccoli B. Time optimal swing-up of the planar pendulum. IEEE Trans. Autom. Control, 2008; 53: 1876-1886.

8. Mikheev YV, Sobolev VA, Fridman EM. Asymptotic analysis of digital control systems. Autom. Rem. Control, 1988; 49: 1175-1180.

9. Reshmin SA, Chernous'ko FL. A time-optimal control synthesis for a nonlinear pendulum. J. Comput. Syst. Sci. Int., 2007; 46: 9-18.

10. Sazhin S, Shakked T, Katoshevski D, Sobolev V. Particle grouping in oscillating flows. Eur. J. Mech. B Fluid, 2008; 27: 131-149.

11. Wang JJ. Simulation studies of inverted pendulum based on PID controllers. Simul. Model. Pract. Theory, 2011; 19: 440-449. 
12. Aström KJ, Furuta K. Swinging up a pendulum by energy control. Automatica, 2000; 36 : 287-295.

13. Chernous'ko FL, Reshmin SA. Time-optimal swing-up feedback control of a pendulum. Nonlinear Dynam, 2007; 47: 65-73.

14. Mason P, Broucke M, Piccoli B. Time optimal swing-up of the planar pendulum. IEEE Trans. Autom. Control, 2008; 53: 1876-1886.

15. Reshmin SA, Chernous'ko FL. A time-optimal control synthesis for a nonlinear pendulum. J. Comput. Syst. Sci. Int., 2007; 46: 9-18.

16. Bloch AM, Leonard NE, Marsden JE. Controlled lagrangians and the stabilization of mechanical systems. I. The first matching theorem. IEEE Trans. Autom. Control, 2000; 45: 2253-2270.

17. Chaturvedi NA, McClamroch NH, Bernstein DS. Stabilization of a 3D axially symmetric pendulum. Automatica, 2008; 44: 2258-2265.

18. Chang LH, Lee AC. Design of nonlinear controller for bi-axial inverted pendulum system. IET Control Theory Appl., 2007; 1: 979-986.

19. Xu C, Yu X. Mathematical model of elastic inverted pendulum control system. Control Theory Technol., 2004; 2: 281-282.

20. Dadfarnia M, Jalili N, Xian B, Dawson DM. A Lyapunov-based piezoelectric controller for flexible cartesian robot manipulators. J. Dyn. Syst. Meas. Control, 2004; 126: 347-358.

21. Dadios EP, Fernandez PS, Williams DJ. Genetic algorithm on line controller for the flexible inverted pendulum problem. J. Adv. Comput. Intell. Intell. Inform., 2006; 10: 155-160.

22. Luo ZH, Guo BZ. Shear force feedback control of a single-link flexible robot with a revolute joint. IEEE Trans. Autom. Control, 1997; 42: 53-65.

23. Tang J, Ren G. Modeling and simulation of a flexible inverted pendulum system. Tsinghua Sci. Technol., 2009; 14(2): 22-26.

24. Krasnosel'skii MA, Pokrovskii AV. Syst. Hysteresis. Springer, Berlin, 1989.

25. Kuwana Y, Shimoyama I, Sayama Y, Miura H. Synthesis of pheromone-oriented emergent behavior of a silkworm moth. Intelligent Robots and Systems'96, IROS 96, Proceedings of the 1996 IEEE/RSJ International Conference on, 1996; 3:1722-1729.

26. Pierce-Shimomura JT, Morse TM, Lockery SR. The fundamental role of pirouettes in Caenorhabditis elegans chemotaxis. J. Neurosci, 1999; 19: 9557-9569.

27. Semenov ME, Solovyov AM, Semenov AM, Gorlov VA, Meleshenko PA. Elastic inverted pendulum under hysteretic nonlinearity in suspension: stabilization and optimal control. COMPDYN 2015 5th ECCOMAS Thematic Conference on Computational Methods in Structural Dynamics and Earthquake Engineering. Eds. M. Papadrakakis, V. Papadopoulos, V. Plevris. Crete Island, Greece, 25-27 May, 2015.

28. Semenov ME, Solovyov AM, Meleshenko PA. Elastic inverted pendulum with backlash in suspension: stabilization problem. Nonlinear Dyn. DOI 10.1007/s11071-015-2186-y. 